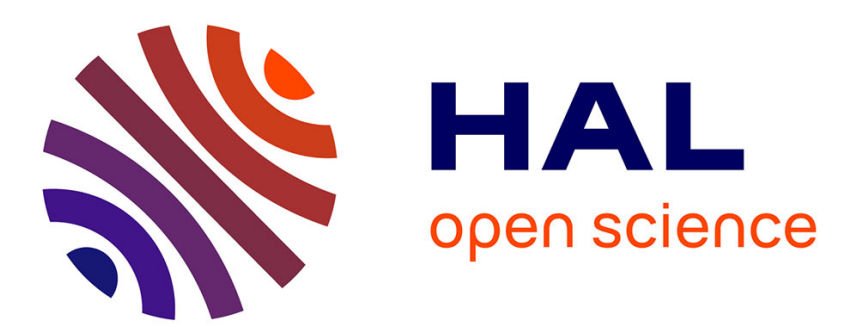

\title{
L'incertaine construction de l'État plurinational et de la démocratie participative en Équateur : aléas et résistances
}

Julie Massal

\section{- To cite this version:}

Julie Massal. L'incertaine construction de l'État plurinational et de la démocratie participative en Équateur : aléas et résistances. Revue internationale de politique comparée, 2013, 20 (1), pp.117-138. 10.3917/ripc.201.0117 . hal-03280760

\section{HAL Id: hal-03280760 https://hal.science/hal-03280760}

Submitted on 7 Jul 2021

HAL is a multi-disciplinary open access archive for the deposit and dissemination of scientific research documents, whether they are published or not. The documents may come from teaching and research institutions in France or abroad, or from public or private research centers.
L'archive ouverte pluridisciplinaire HAL, est destinée au dépôt et à la diffusion de documents scientifiques de niveau recherche, publiés ou non, émanant des établissements d'enseignement et de recherche français ou étrangers, des laboratoires publics ou privés. 


\title{
L'INCERTAINECONSTRUCTIONDEL'ÉTATPLURINATIONAL ET DE LA DÉMOCRATIE PARTICIPATIVE EN ÉQUATEUR : ALÉAS ET RÉSISTANCES
}

\author{
Julie MASSAL
}

Depuis les années 1990 se propage en Amérique latine une vive remise en question du modèle représentatif, et plus généralement de l'insuffisante démocratisation de l'accès au pouvoir politique pour une grande partie des populations indiennes. En effet, dans les pays andins notamment, les acteurs indiens s'organisent et se renforcent, donnant parfois lieu à la formation de ce que Donna L. Van Cott ou Salvador Marti I Puig dénomment les «partis ethniques ${ }^{1}$; et partout, ils remettent en cause les restrictions de l'accès à la citoyenneté. Christian Gros, parmi d'autres, souligne aussi combien en dépit des spécificités, notamment du rythme des réformes et du contexte sociopolitique de chaque pays, cette période s'avère propice aux changements constitutionnels en faveur des droits des peuples autochtones ${ }^{2}$.

Le cas équatorien illustre parfaitement le rôle des acteurs indiens dans la remise en cause des fondements de l'État-nation et de la démocratie représentative et dans la promotion d'un projet politique qui s'inspire du multiculturalisme institutionnel sur certains points, mais s'en distingue sur d'autres ${ }^{3}$. Les organisations indiennes influencent le débat politique à

1. VAN COTT D.-L., (ed), From Movements to Parties in Latin America : the Evolution of Ethnics Politics, Cambridge, Cambridge University Press, 2007 ; MARTI I PUIG S., « Les raisons de l'existence et du succès des partis ethniques en Amérique Latine. Les cas de la Bolivie, de l'Équateur, du Guatemala, du Mexique, du Nicaragua et du Pérou (1990-2005) », in Revue internationale de politique comparée, vol. 17, n², 2010, p. 143-165.

2. GROS C., Nación identidad y violencia : el desafio latinoamericano, Bogotá, UN, IEPRI, U. de los Andes, IFEA, CESO, 2010 ; LE BOT Y., La Grande révolte indienne, Paris, Éditions Robert Laffont, 2009 ; YASHAR D., Contesting Citizenship in Latin America. The Rise of Indigenous Movements and the Post-liberal Challenge, New York, Cambridge University Press, 2007 ; LÉON J.(dir.), Participación política, democracia y movimientos indígenas en los Andes, La Paz, Ambassade de France en Bolivie, IFEA-PIEB, 2005.

3. Le projet indien s'inspire du projet communautarien, tout en reprenant certains thèmes du débat multiculturaliste : MASSAL J., « Movimientos sociales, democracia y multiculturalismo », in MASSAL J., 
maints égards, en promouvant d'importantes réformes sur le plan juridique, constitutionnel et politique, en faveur du pluralisme politique, grâce à leurs solides alliances constituées avec d'autres acteurs sociaux, en faveur d'une réforme de l'État et la démocratie. Ainsi dès 1994, l'option en faveur d'une « participation ${ }^{4}$ des acteurs indiens au pouvoir s'impose, non sans de vifs débats. Mais ce projet politique rencontre de nombreux obstacles, et à partir de 2003, les acteurs indiens, représentés par la Confédération des Nationalités Indiennes d'Équateur (CONAIE) ${ }^{5}$ et le Mouvement Pachakutik, déclinent. C'est pourtant lors du débat de la Constitution en 2008 que leurs principales revendications obtiennent satisfaction.

Quel bilan peut-on tirer de deux décennies de réformes politiques et constitutionnelles et de mobilisation indienne ? L'Équateur a-t-il mis en œuvre une conception multiculturaliste de la démocratie ${ }^{6}$, ou bien s'agit-il de la simple reconnaissance d'un pluralisme restreint qui ne remet pas en cause les fondements de la démocratie libérale ? Notre analyse se fonde sur le travail de terrain effectué pendant notre thèse de doctorat (1997-2001), aussi bien au niveau national que local, puis en 2003 (juillet-août), et sur nos travaux depuis lors. Bien entendu, elle se fonde aussi sur une actualisation bibliographique et un suivi régulier de la presse.

Pour comprendre les enjeux de la reconnaissance du pluralisme politique, tel qu'il se présente dans le cas équatorien, il est nécessaire de montrer comment les acteurs indiens ont su mobiliser autour de leur projet des

BONILLA M., Los movimientos sociales en las democracias andinas, Quito, FLACSO-Ecuador \& IFEA, 2000, p. 157-192 ; MASSAL J., « Los procesos de movilización política de los grupos étnicos en el Ecuador: ¿hacia una sociedad multicultural? », in BONILLA M., MARTINEZ F., SINARDET E., Transformar o reflejar las realidades andinas : la educación en el siglo XX, in Boletín especial del Instituto Francés de Estudios Andinos (IFEA), Lima, vol. 28, n³, 1999, p. 571-611.

4. «Participation » entendue par ces acteurs comme un accès direct et une présence de dirigeants indiens dans les instances de représentation. Toutefois, on peut observer en leur sein de profondes divergences sur la compréhension et les enjeux de cette notion de participation, comme l'illustrent les débats du Second Congrès de Pachakutik (2001) et ceux du bilan de la participation au gouvernement Gutiérrez (septembre 2003), auxquels nous nous référons plus loin.

5. La CONAIE est une organisation nationale qui regroupe trois fédérations régionales : ECUARUNARI, représentative de la Sierra (zone andine), la Confédération Nationale des Indigènes de l'Amazonie Équatorienne (CONFENIAE) qui représente l'Amazonie et à partir de 1990, la Coordination des Organisations Indigènes de la Côte équatorienne (COICE) qui représente la zone côtière. Mais les clivages internes entre Sierra et Amazonie perdurent et ont un impact sur la représentation politique des Indiens.

6. KYMLICKA W., Multicultural Citizenship : a Liberal Theory of Minority Rights, Oxford, Clarendon Press Oxford, 1996. Selon les critères de W. Kymlicka, cela supposerait l'acceptation d'une « citoyenneté différenciée », reliée à des identités culturelles infranationales « minoritaires » et à certains droits collectifs spécifiques. Définition qui présente à la fois des difficultés d'application et de conception théorique et politique dans le contexte andin (car sa conception de l'État plurinational est basée sur le cas des démocraties anglo-saxonnes, notamment canadienne, et suppose une unité politique et culturelle liée à une histoire spécifique, fort différente de celle des pays andins) et des divergences à l'égard des attentes des acteurs indiens eux-mêmes, qui récusent le terme de «minorités ». 
alliés non indiens, durant la décennie $1990^{7}$. On examine ensuite le rapport tissé par les organisations indiennes avec les institutions, rapport marqué par la tension entre conflit et coopération. Il s'agit enfin, à l'issue de ce bilan, de comprendre pourquoi, alors même que les principales revendications indiennes en faveur de la construction de l'État plurinational associé à une démocratie participative semblent obtenir gain de cause, l'un des mouvements indiens les plus forts du continent latino-américain semble durablement marginalisé à la suite d'une brève incursion dans le gouvernement Gutiérrez (2003).

\section{Le projet politique indien : État plurinational et démocratie participative}

Le cas équatorien ayant déjà fait l'objet d'une analyse approfondie auparavant $^{8}$, nous ne reviendrons pas ici sur tous les événements qui ont jalonné la conquête du pouvoir depuis 1990. Il s'agit plutôt d'examiner les relations entre les acteurs indiens et leur environnement politique, qui se caractérisent par une constante tension entre opposition et coopération, même si la seconde prend le dessus entre 1994 et 2003. Rappelons simplement les antécédents majeurs de l'affirmation des acteurs indiens, que sont : le soulèvement de juin $1990^{9}$ lors duquel s'affirme le rôle prépondérant de la CONAIE et surgit la revendication d'une reconnaissance constitutionnelle de l'État plurinational ; puis celui de juin 1994 qui donne lieu à la constitution d'alliances avec des acteurs non indiens, autour de deux principales revendications : la réforme de l'État et la démocratisation de la représentation politique.

On voit ainsi se constituer les deux piliers majeurs du projet politique indien : la reconnaissance constitutionnelle de l'État plurinational et la démocratie participative, comme deux revendications interdépendantes ${ }^{10}$. La notion d'État plurinational suscite de multiples controverses, notamment en avril 1992, lorsque l'Amazonie se soulève à son tour, à travers la marche de l'Organisation des Peuples Indiens de Pastaza (OPIP), revendiquant la reconnaissance des «territoires ancestraux » des peuples indiens : la classe

7. C. Martinez souligne d'ailleurs l'importance des alliances dans la compréhension des mouvements indiens de façon comparée : MARTINEZ C., Repensando los movimientos indígenas, Quito, FLACSOMinisterio de Cultura del Ecuador, 2010.

8. MASSAL J., Les mouvements indiens en Équateur. Mobilisations protestataires et démocratie, Paris et Aix-en-Provence, Karthala-CSPC-IEP, 2005 ; CRUZ E., Movimientos indígenas, identidad y nación en Bolivia y Ecuador : una genealogía del estado plurinacional, Quito, AbyaYala, 2012.

9. MASSAL J., op. cit., 2005 (chapitre 2).

10. MASSAL J., « Mutations et déclin du Mouvement Pachakutik en Équateur (1996-2008) », in Problèmes d'Amérique latine, Paris, Éditions Choiseul, n72, 2009, p. 93-107. 
politique ${ }^{11}$ ainsi que l'armée ${ }^{12}$ y sont foncièrement hostiles. C'est pourquoi une partie des dirigeants de la CONAIE tente d'apaiser le débat et substitue provisoirement au concept d'État plurinational celui d'État pluriculturel, alors jugé moins « subversif », comme on le verra plus loin.

Dès lors, à partir de 1994, c'est la réforme de l'État et de la représentation politique, qui est au cœur du débat. Une nouvelle génération politique, issue de mouvements et partis de gauche affaiblis dans les années 1980, mais aussi des syndicats du secteur public, s'allie à la CONAIE autour de la dénonciation de la mainmise des partis traditionnels sur le pouvoir depuis le retour à la démocratie (1978) ${ }^{13}$. Ainsi, la critique du système politique et électoral jugé peu représentatif progresse et cristallise les alliances autour du projet indien. En outre, la CONAIE prône la relance de la réforme agraire enterrée en $1984^{14}$. À la faveur du soulèvement de juin 1994, elle consolide ses alliances avec les acteurs sociaux non indiens, regroupés dans la Coordination des Mouvements Sociaux (1995) ${ }^{15}$. La CONAIE ne veut plus voir les partis traditionnels représenter les Indiens ; en 1996, on assiste donc à l'émergence du Mouvement d'Unité Plurinationale Nouveau Pays Pachakutik $^{16}$ (MUPNPP) ${ }^{17}$, qui réunit la CONAIE et la Coordination des Mouvements Sociaux (CMS), mais aussi une partie des élites urbaines du milieu culturel et artistique. Celui-ci part à la conquête du pouvoir législatif, et en mai 1996, il devient la troisième force politique du pays avec $10 \%$ des sièges

11. FRANCK E., PATINO N., RODRIGUEZ M., Los Políticos y los Indígenas, Quito, ILDIS, Abya Yala, 1992.

12. ORTIZ C., Indios, militares e imaginarios de nación en el Ecuador del siglo XX, Quito, FLACSOAbya Yala, 2006.

13. Depuis 1979, quatre partis ont dominé la scène politique : le Parti Social-Chrétien, PSC (1951) de tendance droite, le Parti Roldosiste Equatorien, PRE (1982), de tendance « populiste », et deux partis qui furent des acteurs clefs de la transition démocratique (1976-1979), la Gauche Démocratique, ID (1972) et la Démocratie Populaire, DP (1978).

14. La réforme agraire en Équateur est mise en œuvre en 1964 et 1973, puis close par le gouvernement de L. Febrés-Cordero (1984-1988). Initiée par deux gouvernements militaires d'inspiration nationaliste, elle n'a que des effets limités sur la redistribution des terres en zone andine, mais favorise la colonisation des terres en Amazonie. BARSKY O., La reforma agraria ecuatoriana, Quito, CEN, 1988 (2e éd.). 15. Celle-ci regroupe une multitude d'acteurs issus de mouvements sociaux et partis de gauche marxiste affaiblis dans les années quatre-vingt, mais aussi une nouvelle génération de syndicats du secteur public, des organisations féministes, citoyennes, etc. Leur point commun est le refus de la privatisation des ressources naturelles stratégiques et des services publics.

16. Le terme « Pachakutik» d' origine quichua, désigne un concept qui se réfère à une vision du Temps, et il est présenté comme propre à la cosmogonie indienne, selon laquelle un bouleversement profond doit avoir lieu à certaines périodes. Le terme signifie « retour à un ordre nouveau », ce qui implique la conclusion d'un cycle, d'une période définie comme un tout, mais aussi le passage à un temps nouveau. 17. Outre la CONAIE et la CMS, il inclut le Mouvement des Citoyens pour un Nouveau Pays (MCNP) issu de l'Université de Cuenca et représenté par le journaliste F. Ehlers. Mais dès 1997, le mouvement se scinde, avec la sortie du MCNP, et devient le Mouvement d'Unité Plurinationale Pachakutik (MUPP) ou Mouvement Pachakutik. 
au Congrès, tandis que son candidat, F. Ehlers, est quatrième à l'élection présidentielle avec $20 \%$ des voix. Cela augure d'un avenir prometteur pour ce jeune «mouvement politique indépendant », non constitué en parti ${ }^{18}$.

Mais ce succès politique et symbolique indéniable doit être relativisé : le vote pour Pachakutik est peu « indien » dans sa composition sociologique ${ }^{19}$, plutôt urbain et issu de classes moyennes et de milieux intellectuels. De fait, Pachakutik est perçu comme indien par les Blancs-métis et comme métis par les Indiens. Or, le jeu des perceptions compte énormément, dans un contexte dans lequel l'affirmation de l'identité indienne est revêtue d'un enjeu politique majeur et où les définitions en vigueur de l'indianité soulèvent d'âpres débats ${ }^{20}$. Par ailleurs, une grande partie des cadres de Pachakutik au niveau national est «non indienne » et demeure inconnue des communautés indiennes. Au niveau local, les situations varient. Ainsi, il peut exister un mélange de militants indiens ou non indiens, selon le contexte. Par exemple à Otavalo (province Imbabura, au nord de Quito), le mouvement Pachakutik dispose à ses débuts d'une équipe militante jeune, plutôt d'origine urbaine, inexpérimentée et peu liée aux acteurs communautaires ruraux ; ceux-ci, pour leur part, se méfient des militants non indiens ou les connaissent peu. Les dirigeants indiens locaux, comme ils le soulignent eux-mêmes, ont du mal à être identifiés et reconnus dans les communautés indiennes. Ces dirigeants sont, dans ce cas précis, plutôt issus du secteur urbain et artisanal, et éloignés des communautés paysannes. Mais même lorsqu'ils en sont issus, leur « séjour à la ville » durant leurs études, souvent dans des institutions religieuses, les a éloignés à maints égards et leur « réinsertion » dans la communauté d'origine s'avère conflictuelle. Dans ces conditions, Pachakutik a des difficultés à capter le vote indien ${ }^{21}$.

18. La figure du «mouvement indépendant » est autorisée par une loi en janvier 1995. Cependant celuici ne bénéficie pas, contrairement au parti politique, de financement étatique.

19. Une sociologie détaillée de l'électorat pose toutefois une série de problèmes d'ordre méthodologique ; on peut simplement établir des tendances générales, en fonction de la concentration de la population indienne dans certaines provinces. Pour plus de précisions à ce sujet, voir : MASSAL J., op. cit., 2005. 20. Une double remarque d'ordre méthodologique s'impose ici : a) la définition de l'identité indienne implique une analyse des critères utilisés dans les discours officiels (usage d'une langue indienne, appartenance a une communauté indienne), mais aussi du jeu des auto-identifications dans les interactions politiques. Les représentations et les perceptions socioculturelles de ce que signifie «être indien » jouent donc un rôle majeur dans la revendication de cette identité. b) En outre, établir une sociologie proprement dite des acteurs mobilisés, au niveau local, se heurte à de fortes difficultés que ce soit pour obtenir des informations d'ordre personnel ou des données fiables sur le plan quantitatif. Concernant le premier aspect, cela peut s'expliquer par la méfiance persistante des populations indiennes et de leurs dirigeants face à ce qui leur paraît relever de «l'enquête », car les enquêtes « sociologiques » ont traditionnellement été utilisées pour la mise en œuvre de nouveaux impôts dans les communautés. Concernant la détermination quantitative de la population indienne, on rencontre aussi des difficultés en termes de sources et de données disponibles. Le recensement (très contesté) effectué en 2001 donne le chiffre de $7.1 \%$ de population indienne (partant du critère d'auto-identification) et contraste avec des évaluations de chercheurs ( $45 \%$ ), ce qui implique une forte critique des chiffres. Et la détermination démographique et géographique de la répartition de la population indienne pose des défis techniques.

21. Observations de terrain et données issues de nos entretiens à Otavalo, en mai-août 1998. 
En outre, Pachakutik ne parvient pas à construire une base électorale au niveau national ${ }^{22}$ puisque celle-ci reste cantonnée aux grandes villes de la Sierra (zone andine) et à quelques zones de population indienne majoritaire. L'assise territoriale déséquilibrée de Pachakutik sera mise en évidence lors du scrutin présidentiel et législatif de 1998, lorsqu'il régresse pour devenir la cinquième force politique. Ce recul en termes de représentation tient autant aux réformes électorales de 1994-1998, qui favorisent les grands partis traditionnels, qu'aux faiblesses internes de Pachakutik. Ce dernier ne parvient pas à consolider ses bases militantes, et dispose, en tant que mouvement indépendant, de peu de moyens pour faire campagne face aux partis traditionnels qui ont accès aux financements publics. Cette faiblesse ravive les critiques internes à la CONAIE de ceux qui demeurent hostiles à la conquête du pouvoir au niveau national et privilégient l'espace local.

\section{Le rapport aux institutions et l'expérience du pouvoir exécutif}

Ce rapport aux institutions doit être examiné sous trois angles principaux : le premier concerne les relations aux pouvoirs exécutif et législatif ; le second concerne la participation indienne à la formulation de certaines politiques publiques par le biais de différentes instances spécialement consacrées aux « affaires indiennes »; enfin le troisième concerne les relations avec l'armée. Le constat majeur est que le rapport des acteurs indiens au pouvoir est ambivalent, puisqu'il oscille constamment entre la volonté de participation et le rejet ou la contestation, selon les secteurs et acteurs impliqués et selon les opportunités offertes, ou pas, par le gouvernement en place. Cela est d'autant plus marqué après l'expérience de participation au gouvernement Gutierrez (2003) dont l'impact est examiné plus loin.

En premier lieu, revenons sur le rapport au pouvoir. La CONAIE décide de passer de l'opposition et du boycott du système politique et électoral, à la participation au niveau national, après avoir longtemps privilégié l'échelon local et régional, ce qui donne lieu à la constitution du mouvement Pachakutik(1996). Ce en quoi elle se distingue, par exemple, de la mobilisation

22. Il convient de souligner ici deux clivages importants dans la vie politique équatorienne : le clivage Sierra/Costa (zone andine et côtière) qui est déterminant du point de vue électoral pour les acteurs politiques en général ; et le clivage Sierra/Amazonie. Le premier affecte la capacité de Pachakutik à mobiliser sur la Côte, tandis que le second met en évidence les divisions internes aux organisations indiennes. Il faut rappeler aussi que l'Équateur est divisé administrativement en provinces, cantons, paroisses. Les provinces sont représentées par un préfet (élu) et des conseillers provinciaux (élus), les paroisses par un conseil ou assemblée paroissiale (élue) et par une Junte paroissiale (élue), les cantons par des maires et conseils municipaux (élus). La Constitution de 2008 (titre V, chap. 2) apporte quelques modifications, notamment l'introduction des « régions » : http ://pdba.georgetown.edu/Constitutions/ Ecuador/ecuador08.html [consultée le 15 novembre 2011]. 
zapatiste au Mexique (Chiapas), qui se caractérise par un ancrage plus local (au niveau municipal et régional) et un refus de la prise du pouvoir au niveau national ; elle se rapproche en revanche de l'exemple bolivien, où le parti d'E. Morales, le Mouvement vers le Socialisme (MAS), conquiert le pouvoir local, puis législatif et enfin exécutif ${ }^{23}$. Mais cette option participative n'est pas consensuelle au sein de la CONAIE, et n'exclut pas le maintien de la contestation.

En outre il faut clairement distinguer la CONAIE et Pachakutik : la première est issue des « nationalités indiennes » dont elle s'estime le représentant par excellence, et se veut autonome dans ses choix stratégiques, y compris à l'égard de Pachakutik. Ce dernier, en revanche, tout en regroupant la CONAIE et la CMS, représente plutôt une frange de dirigeants sociaux issus des partis d'obédience marxiste et non nécessairement indiens. Or, même si Pachakutik soutient certaines revendications indiennes sur le plan social et politique, il se montre plus réticent face aux demandes d'ordre identitaire, relatives à l'état plurinational, et adopte une attitude plus ambivalente face aux autorités ${ }^{24}$.

Les divergences relatives à la conquête du pouvoir s'accompagnent de tensions permanentes au sujet de la décision de participer au jeu électoral selon les règles en vigueur. Les opposants à la « participation » ${ }^{25}$ arguent de l'impossibilité de changer le système de l'intérieur sans être «corrompu » par celui-ci, et font montre d'une méfiance radicale à l'égard des partis de tous bords qui « instrumentalisent » le vote indien. Cette méfiance persistante inclut les partis de gauche dits « classistes », issus du marxisme, qui valorisent davantage le discours de classe que l'identité culturelle indienne. Pour autant, des intellectuels et membres du secteur universitaire font office d'intermédiaires, en tissant de nouvelles relations avec les jeunes dirigeants indiens. Ceci permet au mouvement Pachakutik naissant de devenir le représentant de divers secteurs sociaux qui aspirent à représenter les « sans voix »

23. DEVINEAU J., «Variations régionales : la politisation des identités ethniques au Mexique », Problèmes d'Amérique latine, Paris, Éditions Choiseul, n72, 2009, p. 73-92 ; DUTERME B., « Quelles lunettes pour des cagoules ? Approches sociologiques de l'utopie zapatiste », in Mouvements, Paris, La Découverte, $\mathrm{n}^{\circ}$ 45-46, 2006/3, p. 107-119. Pour le cas bolivien : CRUZ E., Movimientos indígenas, identidad y nación en Bolivia y Ecuador : una genealogía del estado plurinacional, Quito, AbyaYala, 2012 (en particulier les chapitres 5, 6 et 7 portant sur la période 1990-2008) ; ZEGADA M.-T., La democracia desde los márgenes : transformaciones en el campo político boliviano, La Paz, Ediciones La Muela del Diablo-CLACSO, 2011.

24. Nous nous référons ici à des données récoltées lors d'entretiens sur le terrain, qui mettent en évidence que les dirigeants « classistes » (qui insistent sur le discours de classe) appuient certes la revendication des Indiens d'être mieux représentés politiquement mais pas forcément la plurinationalité, notion dont certains se défient ouvertement.

25. Parfois très radicaux, à tel point que le seul fait de mentionner le terme «participation politique » génère de fortes résistances, comme on peut le constater à Otavalo avec certains membres des organisations indiennes. 
au niveau politique, et non les seuls Indiens. Il s'agit, plus généralement, de renouveler l'offre politique. Le discours de Pachakutik en $1996^{26}$ se fonde principalement sur un renouveau des « façons de faire de la politique ». L'accent est mis sur le retour à des « valeurs morales », tout en insistant sur le « changement radical » devenu indispensable. Mais cet aspect du discours, en outre banal et récurrent chez les « outsiders », perd rapidement de sa crédibilité, car des députés de Pachakutik élus en 1996 sont destitués pour corruption en 1997, ce qui renforce les opposants à la participation électorale. Et surtout, la revendication de pluri-nationalité n'occupe qu'une place discrète, parfois mise en sourdine par certains dirigeants indiens qui la jugent encore peu « audible ${ }^{27}$.

En second lieu, la stratégie conciliatrice de Pachakutik s'avère propice à l'implication croissante des acteurs indiens dans la formulation de certaines politiques publiques via des instances en charge des «Affaires indiennes » au niveau national, à la fin des années quatre-vingt-dix. La progressive insertion de la CONAIE et de Pachakutik dans les instances en question résulte d'une modification de la scène politique. La volonté de Pachakutik d'être partie prenante du jeu politique coïncide avec la volonté d'une partie de l'élite politique d'incorporer les cadres indiens. Les motivations pour ce faire sont diverses : les uns visent à coopter une partie des dirigeants indiens, espérant ainsi les éloigner de la contestation, tandis que d'autres aspirent à faciliter une ouverture de la représentation politique, dans le but de capter le vote indien en leur faveur ${ }^{28}$.

Ces deux motivations guident l'action du présidentA. Bucaram(1996-1997), qui parvient à diviser la CONAIE et Pachakutik. En effet, Bucaram attise les clivages internes de la CONAIE en créant le « ministère des Affaires ethniques », qui coopte une partie des dirigeants indiens, pour l'essentiel formés dans des partis de gauche classistes ou des institutions religieuses, et professionnellement issus de diverses instances liées à l'éducation bilingue. Le secteur andin de la CONAIE dénonce alors les compromissions du secteur amazonien, jugeant « néfaste » la politique gouvernementale, et inversement ${ }^{29}$. En somme, le «ministère ethnique » crée autant de divisions que de nouveaux acteurs prêts à saisir l'opportunité. Et de fait, la « frange andine » apparaît relativement peu crédible dans sa dénonciation,

26. IBARRA H., « Realidad del ajuste define las opciones políticas », in Ecuador Debate, Quito, CAAP, avril 1996, p. 19-28.

27. Comme le souligne L. Macas, haut- dirigeant de la CONAIE : entretiens en septembre 1997, Quito. 28. En effet, les partis traditionnels sont alors en déclin et doivent reconstituer leurs bases électorales. La captation du vote indien, et donc de la région andine (Sierra), s'avère cruciale pour les partis issus de la Costa, tels que le PRE d'Abdala Bucaram, élu en 1996.

29. Ce clivage andin-amazonien recoupe en fait les rivalités entre les deux groupes les plus puissants, les Quichua et les Shuar. Ils sont en compétition pour l'accès aux ressources et au pouvoir. 
puisqu'en 1998, elle change de cap et s'efforce d'intégrer le CODENPE (Conseil de Développement des Nationalités et Peuples d'Équateur, initialement dénommé CONPLADEIN, créé en 1997) ${ }^{30}$, rattaché directement à la Présidence de la République. En effet, la CONAIE, son secteur andin principalement, s'efforce alors de contrôler cet organe, chargé de planifier l'ensemble des politiques publiques en faveur des populations indiennes et qui dispose de financements issus de la coopération internationale. Et ce, en dépit de la rivalité d'autres organisations indiennes comme la Fédération Nationale des Organisations Paysannes Indigènes et Noires (FENOCIN), et la Fédération Évangéliste des Indiens et Noirs d'Équateur (FEINE).

La frange andine, pour justifier son changement d'attitude, argue qu'il s'agit d'une institution distincte des « ministères des Affaires indiennes » créés dans le passé, dotée d'une plus grande légitimité ; en outre sa mission est plus ambitieuse, car il s'agit de coordonner transversalement les politiques publiques ayant un impact sur les populations indiennes. Coordination effectuée par le biais de l'ONG PRODEPINE (Projet de Développement des Peuples Indiens et Noirs d'Équateur), bras exécuteur qui doit répartir les financements aux projets de développement proposés par les communautés indiennes. C'est donc là une opportunité de contrôler ces financements et de permettre à une élite dirigeante indienne, désireuse de se former concrètement, et dont une partie bénéficie de bourses des institutions financières internationales comme la Banque Interaméricaine de Développement (BID), de participer directement à l'élaboration, la formulation et l'évaluation des politiques publiques envers les Indiens. L'enjeu est donc d'importance. C'est aussi pourquoi la CONAIE se radicalise contre L. Gutierrez lorsque celui-ci s'efforce, en 2003, de reprendre le contrôle du CODENPE et de l'ouvrir aux organisations rivales ${ }^{31}$.

Le CODENPE joue un rôle au sein de nombreux projets d'administration publique et territoriale, comme la définition des « circonscriptions territoriales indigènes » reconnues dans la Constitution de 1998. Plus tard il stimulera le débat sur les défaillances du recensement de 2001, et favorisera

30. CODENPE : Consejo de Desarrollo de las Nacionalidades y Pueblos del Ecuador. CONPLADEIN : consejo de desarrollo de los pueblos afrodescendientes e indígenas del Ecuador. Le changement de nom obéit à une mainmise de la CONAIE sur le CODENPE. Précision d'importance, à noter dans le nouveau sigle, l'insistance sur les «nationalités et peuples » : ces « groupes » sont principalement issus des organisations indiennes les plus actives, dans la mesure où « pour être reconnu, un «peuple» doit avoir fait la preuve de sa capacité de mobilisation »; ceci explique le caractère flexible de la liste des peuples et nationalités reconnus par la CONAIE, qui évolue en permanence.

31. En 1998, le Décret Exécutif n³86 (11 décembre) incluait seulement la CONAIE dans son organisation directive : un représentant du gouvernement et un représentant de la CONAIE sont les deux directeurs. Entretiens avec L. Maldonado (CONAIE) et M. Cordova (gouvernement), alors directeurs du CODENPE, Quito, Palais Présidentiel, les 14 et 25 juillet 2000. Aujourd'hui la direction inclut la CONAIE, la FEINE et la FENOCIN. Voir http ://www.codenpe.gov.ec/ [consultée le 24-02- 2012]. 
avec d'autres instances publiques en charge des statistiques (INEC, SIISE) la réflexion sur les modalités du recensement de 2011 ${ }^{32}$. Avec PRODEPINE, il est le principal organe de mise en œuvre des politiques de développement en faveur des communautés. Son bilan s'avère pourtant mitigé sur ce plan, et, on le verra, cela influe négativement sur la mobilisation. Outre le CODENPE, les acteurs indiens disposent de différentes instances, comme la Direction de l'Éducation Bilingue Interculturelle (DINEIB), ou le Fonds de Développement des Nationalités et Peuples Indiens d'Équateur (FODEPI) ${ }^{33}$, qui bénéficient de financements publics, mais dont les marges d'action sont restreintes. Ainsi, malgré des progrès indéniables de la présence indienne au sommet de l'État, l'effet sur le développement des populations indiennes reste mitigé.

En troisième lieu, il convient de mentionner le lien ambigu qui relie les acteurs sociaux, notamment indiens, aux militaires. Ainsi, le coup d'État du 21 janvier 2000 met en évidence une alliance entre la CONAIE et les officiers de l'armée de terre conduits par le colonel L. Gutiérrez. Mais si les insurgés obtiennent le départ du président J. Mahuad (1998-2000), le pouvoir revient au vice-président, G. Noboa (2000-2003) soutenu par la hiérarchie militaire ${ }^{34}$. L'alliance entre la CONAIE et l'armée de terre s'avère insolite à maints égards, au vu de leurs divergences au sujet de l'État plurinational et des autonomies indiennes; deux revendications fondamentales de la CONAIE auxquelles l'armée est résolument hostile. Il faut toutefois rappeler que les acteurs indiens et l'armée se rejoignent sur deux autres discours majeurs : la défense des « ressources naturelles stratégiques », notamment le pétrole, en Amazonie, en grande partie contrôlé par l'armée face aux privatisations, et le refus des autonomies régionales promues par certaines provinces mieux dotées en ressources, certaines menaçant, au moment où se réalise le coup d'État, de faire sécession. Mais l'alliance entre la CONAIE et l'armée est de courte durée : les divergences l'emportent.

Un autre impact du coup d'État est l'affrontement de la CONAIE avec Pachakutik : la première demande aux députés Pachakutik élus en 1998 de démissionner et qui refusent. La direction de Pachakutik dénonce le coup d'État et continue de promouvoir la conquête du pouvoir par la voie électorale. Ainsi les deux entités divergent dans leur rapport au pouvoir. Par la suite, Pachakutik appuie la candidature présidentielle de L. Gutiérrez

32. RADCLIFFE S.A., «Re-Mapping the Nation : Cartography, Geographical Knowledge and Ecuadorean Multiculturalism », in Journal of Latin American Studies, Cambridge, Cambridge University Press, n42, 2010, p 293-323.

33. Le FODEPI (http ://www.fodepi.gov.ec/) s'autodéfinit comme une entité publique au service du développement des nationalités et peuples indigènes. Il exerce des missions de formation et de financement auprès des communautés indiennes.

34. Sur ces événements : MASSAL J., op. cit., 2005, chap. 8. 
tandis que la CONAIE se montre réticente. En effet, à l'automne 2002, c'est Pachakutik qui décide, le premier, de faire alliance avec L. Gutiérrez, amnistié et devenu candidat à l'élection présidentielle avec le soutien de son parti, Société Patriotique. La CONAIE ne se rallie à Gutiérrez qu'après son échec à imposer son propre candidat, en raison de ses dissensions internes.

Élu grâce aux organisations indiennes, L. Gutiérrez ouvre son gouvernement à six ministres issus de Pachakutik, en janvier 2003. Le rapport des acteurs indiens au gouvernement Gutiérrez s'avère cependant très vite conflictuel : Pachakutik est confronté à un dilemme puisque la politique présidentielle va à l'encontre des promesses de campagne et le place ainsi en porte-à-faux à l'égard de ses bases. La CONAIE retire son soutien, et Pachakutik, rapidement affaibli par les démissions de ses ministres, sort finalement du gouvernement contraint et forcé (6 août 2003) ${ }^{35}$.

L'expérience de «participation au pouvoir », alors jugée historique se révèle donc être un fiasco, dont les effets se font encore sentir dix ans plus tard. Les répercussions sont principalement le déclin de la mobilisation indienne, mais aussi celui des alliances et la perte de crédibilité du projet indien ; il s'ensuit un fort déclin électoral de Pachakutik. L'affaiblissement de la mobilisation obéit à divers motifs ${ }^{36}$. D'une part, les acteurs indiens incarnés par la CONAIE sont de plus en plus isolés. Gutiérrez avive des clivages locaux ou régionaux et stimule la concurrence entre les organisations indiennes, la FENOCIN et la FEINE devenant alors les nouveaux interlocuteurs du gouvernement. D'autre part, les six mois de présence indienne au gouvernement ont produit peu de résultats sur les politiques publiques en faveur des populations indiennes, faute de temps et de moyens, mais ont un impact retentissant et néfaste sur la légitimité et la capacité de mobilisation de la CONAIE et du mouvement Pachakutik. Toutefois ce discrédit ne tient pas seulement à l'échec gouvernemental de 2003, mais aussi aux tensions internes qui deviennent de plus en plus évidentes et au divorce croissant avec les bases.

En effet, la CONAIE est durablement discréditée auprès des communautés indiennes et parvient donc de moins en moins à les mobiliser. Dans le même temps, celles-ci sont à la fois « cooptées » par les politiques clientélistes du gouvernement (celui-ci distribue du matériel agricole : « politique »

35. Pour plus de précisions : MASSAL J., « La participation du Mouvement Pachakutik au pouvoir en 2003 : une expérience originale de citoyenneté multiculturelle ? », in BLANQUER J.-M. et al., Voter dans les Amériques, Paris III-Sorbonne Nouvelle-IHEAL-Institut des Amériques, 2005, p. 263-272. Voir aussi : MUNOZ J.O., Organizaciones indígenas ecuatorianas, Madrid, U. Complutense, 2009.

36. Pachakutik réalise un bilan critique de sa «participation » au pouvoir durant son Congrès en septembre 2003 : le rapport du Congrès retrace la vision du mouvement sur les années 2000-2003. Il est disponible sur http ://www.llacta.org/organiz/coms/com368.htm [Consulté le 01-12-11]. 
connue comme celle « des pelles et des pioches »), et de plus en plus absorbées par l'élaboration de leurs « projets de développement » pour obtenir les financements du CODENPE. Ce second aspect est d'ailleurs analysé par V. Breton comme un élément majeur du déclin des acteurs indiens : les communautés, de plus en plus soucieuse d'obtenir des financements, finissent par passer d'un « projet » à l'autre ; or, ces projets en série sont de courte durée et de faible portée, et ont peu d'impact à long terme sur le développement économique des communautés, mais « absorbent les énergies » des individus et les détournent de la contestation ${ }^{37}$. Pour sa part, L. Tuaza analyse ce déclin de la mobilisation des communautés comme le fruit d'une « fatigue organisatrice » de plus en plus prégnante, et d'un divorce croissant entre elles et les dirigeants de la CONAIE, perçus comme des acteurs surtout soucieux de leurs « intérêts propres » et finalement peu rénovateurs en termes de pratiques politiques ${ }^{38}$. Tuaza souligne également les limites des projets de développement, et leurs effets délétères sur les communautés, qui se déchirent autour de l'obtention de financements ponctuels. Ainsi la CONAIE perd-elle ses bases dans les régions à majorité indienne (provinces Imbabura, Bolivar et Chimborazo), auparavant lieux historiques de mobilisation. Et, dix ans après l'expérience de «participation », cet affaiblissement persiste.

À partir de 2003, la CONAIE perd également le soutien de ses alliés non indiens, heurtés par son « ethnicisation » à outrance : une partie des dirigeants valorisent « les façons indiennes spécifiques » de faire de la politique, en opposition aux pratiques « occidentales » considérées comme amorales, et développent parfois aussi un certain racisme envers les non-Indiens. Les alliés sont rebutés par cette tendance au repli sur soi et à l'autoreprésentation, que traduit le slogan « seuls les Indiens peuvent représenter les Indiens ». Cela mine considérablement Pachakutik, dont l'un des principaux appuis électoraux était le vote urbain blanc métis. Par ailleurs, le principe de renouvellement de l'offre politique n' apparaît plus très crédible, à la fois dans les communautés et dans la population en général : le caractère novateur, le discours de rupture et la dénonciation de la corruption perdent leur capacité d'attraction. Enfin, des voix s'élèvent contre l'incapacité de Pachakutik à profiter de cette «opportunité historique », et contre la préférence de la CONAIE pour « l'opposition systématique ». En somme, on assiste à la

37. BRETON V., «La deriva identitaria del movimiento indígena en los Andes ecuatorianos o los límites de la etnofagia », in MARTINEZ C., (ed.), Repensando los movimientos indígenas, Quito, FLACSOMinisterio de Cultura, 2010, p. 69-121. On peut aussi consulter : MORICEAU C., «Agents de développement et militants paysans indigènes dans les Andes équatoriennes. La quête du juste équilibre face aux préceptes de la coopération internationale », in Mémoire pour le Master Recherche Sociologie et Institutions du Politique, Département de Science politique, Université Panthéon-Sorbonne, 2011-2012. 38. TUAZA L.A, La crisis del movimiento indígena, Quito, FLACSO, 2011. Nous avions aussi réalisé cette observation en 1998 à Otavalo auprès de dirigeants communautaires. 
marginalisation politique, mais aussi discursive et symbolique des acteurs indiens, encore sous le choc de leur éviction du gouvernement Gutiérrez ${ }^{39}$.

Sur le plan électoral, la situation est plus critique encore. Entre 2004 et 2006, la CONAIE et Pachakutik sont en position de repli, ce qui se traduit par leur incapacité à proposer un candidat indien consensuel. Cela met aussi en exergue le divorce existant au sein même de la CONAIE, dont les acteurs locaux contestent les décisions prises au niveau national. Après maintes tergiversations sur l'éventuelle alliance avec Rafaël Correa, alors candidat à la présidentielle, du fait des divisions de la CONAIE et Pachakutik concernant la stratégie électorale ${ }^{40}$, Pachakutik décide de se lancer seul à la présidentielle en 2006 ; son candidat, très contesté par les organisations locales et les bases, est le leader historique Luis Macas (en dépit de son expérience malencontreuse au gouvernement Gutiérrez). Cette situation atteste du manque de renouvellement des cadres dirigeants, ce qui motive également de vives critiques des militants locaux. Et face à la défaite électorale en 2006, lorsque Macas n'obtient que $2.1 \%$ des votes, c'est l'option même de la participation électorale qui est remise en cause. Le mouvement Pachakutik se fracture, certains de ses cadres rejoignant R. Correa. Ce dernier, ministre de l'Économie durant le gouvernement intérimaire d'A. Palacios (avril 2005-décembre 2006) ${ }^{41}$, remporte l'élection présidentielle et reprend à son compte le discours « antisystème », notamment en promettant de renforcer le contrôle de l'État, via l'accroissement des impôts, sur les multinationales qui exploitent les hydrocarbures, en refusant le paiement de la dette extérieure et en promouvant la dissolution du Congrès, très décrédibilisé. Correa capte alors le vote contestataire qui s'était orienté auparavant vers Pachakutik.

Ainsi, en 2006, la mobilisation électorale et la mobilisation sociale sont durablement affaiblies. Ce qui oblige les acteurs indiens à une remise en question interne et à un renouvellement de leur projet. Pourtant, dans le même temps, la société équatorienne semble devenir plus réceptive au discours indien. Le contenu et l'influence de ce discours doivent donc être évalués à l'aune des événements retracés jusqu'ici. Ceci permet de comprendre le contraste entre les changements majeurs obtenus sur le plan constitutionnel, et le déclin politique persistant des acteurs indiens depuis une décennie.

39. Les entretiens réalisés avec des dirigeants indiens et non indiens de la CONAIE et Pachakutik illustrent ce traumatisme dû au sentiment de « trahison du mouvement indien » (la CONAIE) par L. Gutiérrez. Entretiens avec V. Hernandez, ex-vice-premier ministre ; I. Narvaez, représentant syndicaliste membre de Pachakutik, et divers leaders de la CONAIE, Quito, août 2003.

40. Un exemple de ces divisions est celui d'Otavalo, où la présidente de la Fédération Indigène et Paysanne d'Imbabura (FICI), antenne locale de la CONAIE, refuse d'appuyer le mouvement Pachakutik. Les divisions portent sur la stratégie électorale en 2004 puis sur le choix du candidat Pachakutik aux élections présidentielles de 2006.

41. A. Palacios succède à L. Gutiérrez (2003-2005), démis par le Congrès lors de la « Révolution des Forajidos ». La CONAIE ne prenant qu'une part marginale à ces évènements, nous ne les développons pas dans cet article. 


\section{Le lien entre état plurinational et démocratie participative}

Dans la décennie 1998-2008, deux constitutions sont débattues et promulguées, et offrent ainsi un certain nombre de changements, en termes de reconnaissance du pluralisme politique, de l'ouverture de la représentation politique et de modification des rapports sociaux et politiques entre acteurs indiens et blanc métis, le cas équatorien s'inscrivant ainsi dans une tendance plus large en Amérique andine ${ }^{42}$. Si les modifications constitutionnelles restent parfois lettre morte dans les faits, leur dimension emblématique n'en est pas moins indéniable, puisque c'est le fondement même de la construction de la Nation qui est en jeu.

Lors du débat des constitutions, via les Assemblées constituantes, les acteurs indiens s'efforcent d'occuper l'espace public en proposant leur propre projet constitutionnel. Le « discours indien », constitué par les positions de la CONAIE et de Pachakutik, sans occulter leurs divergences stratégiques parfois profondes, se fonde depuis 1990 sur quatre thèmes majeurs : l'État plurinational, la démocratie participative, la décentralisation, et les droits collectifs ${ }^{43}$. L'élément le plus significatif est le lien étroit établi entre «État plurinational et démocratie participative ». Les droits collectifs et la décentralisation en découlent. Rappelons toutefois que l'usage fait par les acteurs de certaines notions, telles que peuple ou nationalité, ne recoupe pas les concepts de la littérature scientifique sur le nationalisme ou les droits des minorités ${ }^{44}$. Raison pour laquelle nous nous intéressons ici à « la façon dont les acteurs définissent ces notions et les emploient », plutôt qu'à leur « conformité » avec la théorie politique.

Les Constitutions de 1998 et 2008 ont contribué à l'accroissement des droits des Indiens, mais s'inscrivent dans un contexte différent ${ }^{45}$. L'Assemblée

42. VAN COTT D.L, « Cambio institucional y partidos étnicos en Suramérica », in Análisis Político, Bogota, IEPRI-UN, n ${ }^{4} 8,2003$, p. 26-51 ; MASSAL J., « Cambio constitucional y democracia », in Análisis Político, Bogotá, IEPRI-UN, n75, 2012, p. 29-46. Ces articles analysent, à partir d'études de cas andins, le lien entre changement constitutionnel et approfondissement de la démocratie. Le premier analyse notamment les cas colombien et bolivien, et le second est centré sur les cas équatorien et bolivien. 43. Si la littérature scientifique sur les notions d'État pluri/multi culturel, ethnique et plurinational a permis une analyse rigoureuse de ces réalités politiques, elle présente l'inconvénient, comme nous l'avons montré ailleurs, de se rattacher à l'étude des sociétés européennes et anglo-saxonnes. Or le fondement de la construction de l'État-Nation est historiquement distinct dans les pays andins : MASSAL J., « Los procesos de movilización política de los grupos étnicos en el Ecuador :¿hacia una sociedad multicultural? », in BONILLA M., MARTINEZ F., SINARDET E., op. cit., 1999. Sur le discours indien, voir les Projets politiques de la CONAIE et ceux de réforme constitutionnelle en 1997 et 2007.

44. MASSAL J., op. cit., 2005.Nous analysons en détail ces notions, leur usage par les acteurs, et leur relation avec la théorie politique, au chapitre 7 et en conclusion. Voir aussi : PNUD, Etnicidad y Ciudadanía, Programa de las Naciones Unidas para el Desarrollo, Guatemala, 2009 [coordinador : Roddy Brett] : http ://www.pnud.org.gt/data/publicacion/Etnicidad_y_ciudadania.pdf [consulté le 13 décembre 2012]. 45. Les constitutions sont disponibles en anglais et espagnol : Base de données, Université Georgetown : http ://pdba.georgetown.edu/Constitutions/Ecuador/ecuador08.html http ://pdba.georgetown. edu/Constitutions/Ecuador/ecuador98.html [Consultées le 15 novembre 2011]. 
constituante de 1997-1998 se focalise sur l'instabilité politique, interprétée en termes de crise de « gouvernabilité » : les réformes se concentrent sur les changements institutionnels, électoraux, et économiques ${ }^{46}$. La CONAIE et ses alliés orientent leurs efforts vers la reconnaissance de la pluri-nationalité, mais n'obtiennent que celle de l'État pluriculturel et multiethnique (article 1). Il convient en effet de distinguer l'État pluriculturel et l'État plurinational : le premier sous-entend la diversité des cultures dans l'unité politique nationale, tandis que le second renvoie à une conception critique de l'État-nation, construit sur le «mythe » de l'unité culturelle et le métissage ${ }^{47}$. La pluri-nationalité suppose une transcription politique de la diversité culturelle et implique une réorganisation des fondements sociaux, juridiques, économiques et culturels en faveur des peuples indiens reconnus comme une entité politique. Au niveau politique cela implique une représentation des « peuples » sur la base de leur identité culturelle. Le terme d'État multi/pluriethnique, pour sa part, renvoie à une conception de la diversité en termes d'ethnies, à l'origine d'âpres débats puisque les acteurs indiens refusent d'être définis comme des minorités ethniques.

La Constitution de Riobamba (1998) ne reprend pas la proposition de la CONAIE d'instaurer la « représentation des nationalités et peuples indiens via un système de quotas »; par contre elle incorpore la reconnaissance des « circonscriptions territoriales indigènes »; thème qui, lui, est absent du projet de la CONAIE. Elle illustre ainsi la tendance des acteurs non indiens à circonscrire les acteurs indiens à des enclaves territoriales strictement délimitées ; cela va à l'encontre du projet de la pluri-nationalité qui repense au contraire l'ensemble de l'organisation politique et sociale nationale. Cela montre que la reconnaissance des droits collectifs des « peuples indiens qui s'autodéfinissent comme des nationalités aux racines ancestrales » (art. 83), ne suffit pas à l'instauration de l'État plurinational ${ }^{48}$. C'est toutefois l'avancée majeure en 1998 : ces droits, énumérés à l'article 84 et au nombre de 15 , concernent aussi bien l'organisation interne des peuples et communautés que les relations de ceux-ci avec les acteurs externes, au sujet notamment de l'exploitation des ressources du sous-sol par l'État. Toutefois, la mise en application légale tarde et la réalité montre la faible prise en compte de ces droits. Ce débat est donc réactivé dix ans plus tard.

46. Voir le dossier sur la Constituante de 2007-2008 et les analyses rétrospectives sur 1997-1998 de l'Institut de Recherche et Débat sur la Gouvernance (Bogotá) [Consultés les 15, 16 et 23 novembre 2011] : http ://www.institut-gouvernance.org/fr/synthese/fiche-synthese-20.html

47. VERDO G., VIDAL D., «L'ethnicité en Amérique latine : un approfondissement du répertoire démocratique ? », in Critique internationale, Paris, CERI-FNSP, n57, 2012, p. 9-22. L’article analyse notamment les antécédents historiques et la notion d'État-nation et de métissage dans les pays latinoaméricains.

48. La reconnaissance de «peuples qui s'autodéfinissent comme des nationalités » (art. 84) dotés de droits collectifs est considérée comme un premier pas vers la plurinationalité, mais cela reste insuffisant aux yeux de la CONAIE, car ces peuples ne sont pas dotés d'une capacité de représentation politique autonome. 
Le contexte diffère en 2007-2008 : le gouvernement Correa, dont le parti Alliance Pays (AP) détient 80 des 130 sièges à l'Assemblée constituante, est soutenu par une frange de Pachakutik, car il promet une prise en compte des revendications indiennes. Mais l'incompréhension ou la méconnaissance autour des notions de pluriculturalité et pluri-nationalité persiste ${ }^{49}$. La Constitution de Montecristi (2008) est innovante à plus d'un titre. En effet, elle s'inspire des principes du «Sumak Kawsai » («le Bien-Vivre ») ${ }^{50}$, qui suppose une réforme de la conception du développement économique, la reconnaissance des droits de la nature et notamment de l'eau comme bien commun inaliénable, et implique une série de changements dans les conditions de vie des peuples indiens ${ }^{51}$. L'État plurinational et interculturel est reconnu (art. $1^{\mathrm{er}}$ ), de même que l'usage des langues indiennes Quichua et Shuar comme « langues officielles de relation interculturelle », tandis que les autres langues indiennes ne sont que « des langues d'usage officiel» dans les territoires où elles sont «utilisées dans les termes que fixe la loi » (art. 2). Les groupes indiens dénommés peuples voient également leurs droits collectifs étendus (article 57, titre II, chapitre 4). Désormais ils sont au nombre de 21, puisque de nouveaux droits sont introduits tandis que d'autres sont approfondis. Ainsi, les principales modifications par rapport à 1998 sont : la « consultation préalable libre et informée » sur les projets d'exploitation de ressources naturelles (al. 7), « le rejet du racisme et de toute forme de discrimination » (al. 2), « le maintien et la protection des savoirs ancestraux collectifs et des ressources génétiques qui supportent la diversité biologique et l'agrobiodiversité [...]. L'interdiction de toute forme d'appropriation de leurs connaissances, innovations et pratiques » (al. 12) ou « l'interdiction de tout type d'activité extractive dans les territoires des peuples en situation d'isolement volontaire » (al. 21). Les changements introduits en 2008 s'inscrivent dans un contexte marqué à la fois par l'affaiblissement de la

49. Sara Vogel examine la diversité des positions : certains promeuvent l'interculturalité, d'autres la plurinationalité (parfois jugées incompatibles). Le texte final reconnaît à la fois l'État interculturel et plurinational : VOGEL S., «Los Alcances y Límites de Participación Ciudadana : la Conaie y la plurinacionalidad en la Asamblea Constituyente de 2008 », in ISP Collection, 2008, Paper 33 : http :// digitalcollections.sit.edu/isp_collection/33 [Consulté le 10 novembre 2011].

50. Une notion de plus en plus débattue par différents courants des partis et intellectuels issus de la gauche (comme A. Borón, E. Gudynas), qui prétend allier le contrôle national (et le refus de la privatisation) des ressources naturelles stratégiques, à la prise en compte d'éléments du discours sur le « développement durable » et à certains aspects du discours indien sur la Terre-Mère (la Pachamama). Pour un bilan, voir le texte promulgué par le gouvernement Correa et le Secrétariat National pour la Planification du Développement (SENPLADES) : «Plan national pour le Bien-Vivre. Construire un État Plurinational et Interculturel (2009-2013) », Quito, SENPLADES, 2009, dont l'introduction retrace une vision historique de la notion de Bien-Vivre.

51. Voir : «Plan national pour le Bien-Vivre. Construire un État Plurinational et Interculturel (2009-2013)», op.cit., qui expose les propositions gouvernementales pour mettre en application la Constitution. Et le « Plan plurinational pour éliminer la discrimination raciale et l'exclusion ethnique et culturelle (2009-2012) », $2^{e}$ éd., 2010 (pdf) ; texte gouvernemental qui met en œuvre une série de politiques publiques contre la discrimination. 
CONAIE et Pachakutik et par le renouveau d'un discours étatique et centré sur l'État-nation souverain, porté par R. Correa. La Constitution porte la marque du projet présidentiel, soucieux de revaloriser le rôle de l'État dans l'économie et de renforcer la souveraineté nationale. Toutefois, elle incorpore des réformes qui répondent aux attentes majeures des acteurs indiens surtout en ce qui concerne la protection de la nature et des ressources stratégiques, et revalorise les droits collectifs.

Une synthèse s'opère grâce à Monica Chují, présidente de la $5^{\mathrm{e}}$ table sur les ressources naturelles durant l'Assemblée constituante et auparavant secrétaire générale de communication de la Présidence de la République du gouvernement Correa. Cette dirigeante indienne issue de l'Amazonie aspire à déconstruire les «mythes » qui entourent la pluri-nationalité, en publiant un texte en plein cœur de l'Assemblée constituante ${ }^{52}$. Elle défend la thèse de la complémentarité entre pluri-nationalité et interculturalité tout en montrant que la première va plus loin et s'avère indispensable pour une démocratie effective : «L'État plurinational est la condition qui rend possible l'enrichissement de la démocratie avec l'apport et l'expérience des peuples et nationalités ». Et elle ajoute : «L'Équateur se trouve à un moment crucial de son histoire. S'il reconnaît la pluri-nationalité [...], il aura adopté un chemin de décolonisation de la démocratie et de construction d'un horizon à long terme dans lequel toutes les volontés peuvent construire l'unité dans la diversité ». Ainsi, la pluri-nationalité ne signifie ni démembrement ni séparatisme, comme le suggèrent ses détracteurs, mais implique une cohabitation et un échange respectueux de différentes cultures, non pour le métissage et l'assimilation, mais pour le respect de la diversité culturelle, au sein de l'unité nationale. Et l'État plurinational devient la condition même d'une démocratie « décolonisée », débarrassée des « traits racistes et discriminants » qui sont au fondement de l'État-nation.

Le lien entre État plurinational et démocratie mérite d'être approfondi. Dans le discours indien, notamment celui de Pachakutik, la démocratie doit être « locale ou directe », et favoriser une plus grande « participation » des peuples indiens reconnus en tant que tels et dotés de droits collectifs, ainsi qu'une meilleure qualité de la « représentation ». Le discours tend pourtant à varier selon le contexte et le rapport au pouvoir : la définition et la portée de la démocratie participative, et ce que recouvre la notion même de participation, se modifient. Ainsi, dans le second congrès de Pachakutik en septembre 2001, l'accent porte sur le renforcement du pouvoir local et l'engagement des acteurs à travers différents mécanismes participatifs,

52. M. Chují, « Dix concepts basiques sur la plurinationalité et l'interculturalité », Montecristi, 9 avril 2008. Source : Agencia Latinoamericana de Información (ALAI) http ://alainet.org/active/23366 (en espagnol). [Consulté le 11 novembre 2011]. 
assemblées et consultations. La décentralisation devient ainsi l'instrument d'une « démocratie par le bas ». À partir de 2003, suite à l'échec de la «participation au gouvernement », ici entendue comme présence de dirigeants indiens en son sein, le mouvement Pachakutik tend à renforcer l'accent mis sur l'espace local de représentation et participation, et à promouvoir l'usage de divers " dispositifs participatifs », certains spécifiques aux peuples indiens, et d'autres issus des organes représentatifs municipaux ou cantonaux ${ }^{53}$. L'on passe ainsi, dans le discours indien, d'une participation conçue comme une simple présence au sein des organes de pouvoir nationaux, exécutif ou législatif, à un investissement dans un certain nombre d'espaces locaux, eux-mêmes en transformation du point de vue de leur organisation politique et électorale. Ce qui illustre combien les usages et la notion même de «participation » varient notoirement dans le discours des acteurs indiens, selon qu'ils sont ou non en position de force.

La revalorisation du pouvoir local se traduit aussi par la multiplication d'assemblées législatives dénommées « congrès des peuples » : ces derniers sont censés promouvoir la délibération et la consultation politique desdits peuples et faciliter leur accès au débat politique. D'autres modalités de « participation » existent, à travers les gouvernements communautaires ou « cabildos », organes exécutifs et délibératifs qui représentent la communauté ${ }^{54}$ et la consultent via les assemblées internes, ou bien des mécanismes comme ceux mis en œuvre à l'échelle cantonale à Saquisili ${ }^{55}$; ou encore des expériences de budget participatif, de radios locales, etc. En bref, tout un processus de réorganisation sociale au niveau cantonal et municipal, qui illustre le repli sur l'espace local. Si le bilan de ces différentes formes de participation et de leur impact s'avère complexe, car parcouru par des tensions et dynamiques locales contradictoires, une tendance récurrente depuis 1998 est la « revitalisation culturelle » : engagée par les cabildos communautaires avec des soutiens externes (ONG, centres de recherche, universités), cette revitalisation conduit à un processus de « réinvention de la tradition », selon l'expression d'Hobsbawm et Ranger ${ }^{56}$, et participe de la consolidation des gouvernements communautaires autonomes.

53. Pour plus de détails à ce sujet : MAZAR N., « Mouvement indigène en Équateur, l'expérience de participation à la gestion du pouvoir : pratiques communautaires et principe participatif », Mémoire de Master en Études des sociétés latino-américaines, Paris, U. Paris III-IHEAL, 2005 ; LE QUANG M., «La gestion politique dans le canton de Saquisili en Équateur : entre démocratie participative et 'corporatisme indigène' », communication aux journées doctorales du GIS, Participation du public, décision, démocratie participative, ENS-LSH, Lyon, 27-28 novembre 2009 (pdf, 15 p.).

54. Les communautés indiennes jouissent d'un statut spécifique (1967) qui leur permet de se doter de leurs propres autorités locales, mais qui évolue à la fin des années 1990, l'objectif étant de reconstruire des « gouvernements communautaires » consolidés et autonomes : MASSAL J., op. cit., 2005.chap. 7. 55. LE QUANG M., op. cit., 2009.

56. HOBSBAWM E., RANGER T.O., (eds), The Invention of Tradition, Cambridge, Cambridge University Press, 1983. 
Au niveau national, la démocratie doit, selon le discours indien, se renforcer grâce au déploiement de l'État plurinational dans la vie sociale, politique et juridique, afin de rompre avec un passé colonial encore trop présent. Mais de nombreux projets demeurent encore incertains, comme la délimitation administrative des circonscriptions territoriales indigènes, au sein de l'agencement territorial lui-même modifié en 2008, et surtout, la mise en œuvre effective des droits collectifs. Parmi ceux-ci, le plus débattu est le droit à la « consultation préalable, libre et informée » des communautés sur d'éventuels projets d'exploitation des ressources du sol et du soussol. En effet, cela revêt une importance stratégique comme Chují (2008) le souligne, puisqu'il s'agit à terme d'octroyer le droit, "non seulement aux Indiens, mais à tous les Équatoriens », de se prononcer sur les projets d'exploitation des ressources, et ce « sur l'ensemble du territoire national », pas seulement dans les territoires indiens. En cela, Chují illustre l'idée selon laquelle la défense de l'État plurinational et des droits collectifs devient un moyen de lutte pour la « défense du patrimoine national », contre l'appropriation privée des ressources naturelles. En outre, la pluri-nationalité ne vise pas à créer un droit parallèle pour les seuls Indiens, mais incite bien au contraire à l'extension des droits collectifs à l'ensemble de la population ${ }^{57}$. Dès lors, la pluri-nationalité signifierait la mise en œuvre d'un « nouvel ordre politique et économique » favorisant le pouvoir des peuples. Il faut noter que dès 1998 des groupes « non indiens » cherchent à se faire reconnaître sous la forme de «peuples » pour accéder aux droits collectifs.

On comprend mieux pourquoi la revendication d'État plurinational suscite encore une forte hostilité en haut lieu, exprimée par R. Correa entre 2006 et 2008 dans ses discours publics, lorsqu'il dénonce tout à la fois « la plurinationalité, l'anarchie » promue par les mouvements sociaux et « l'opposition irresponsable » à l'exploitation minière des « $2 \%$ », chiffre qui fait allusion au résultat du candidat de Pachakutik, Luis Macas, à l'élection présidentielle en 2006. En témoigne le discours présidentiel du 27 mars 2008 au sujet de la pluri-nationalité, que Correa définit comme « une alliance de territoires » où « [...] [les secteurs radicaux] prétendent commander » [au lieu du] « légitime gouvernement de l'État équatorien » ${ }^{58}$. L'ambiguïté présidentielle à ce sujet montre bien que la revendication heurte son propre projet politique et économique ; les droits collectifs restent lettre morte, quand l'État se heurte au refus des communautés indiennes de permettre l'exploitation des ressources stratégiques, comme l'illustrent les mobilisations de communautés d'Amazonie en septembre 2009 contre l'exploitation

57. MASSAL J., « Citoyenneté ethnique, droits collectifs et décentralisation dans les Andes : des outils de reconstruction de l'État-nation ? », in COHEN J., SPENSKY M., Citoyenneté et diversité, ClermontFerrand, Ed. PUF-Université Blaise Pascal, 2009, p. 229-257.

58. OSPINA P., «El Ecuador de Rafael Correa », in Quito, CEP, mai 2008 (pdf, 20 p.). La citation issue du discours présidentiel du 27 mars 2008, est faite par l'auteur p. 16 (Citation traduite). 
minière. À compter de cette date, la rupture entre les acteurs indiens et Correa est flagrante : la CONAIE et Pachakutik dénoncent les arrestations de leurs dirigeants et militants ; le président, en retour, n'a de cesse de dénier toute légitimité et représentativité à Pachakutik, affaibli électoralement et décrédibilisé depuis $2003^{59}$. Le déclin persistant, sur le plan social et politique, de l'un des mouvements indiens longtemps considéré parmi les plus solides en Amérique latine, s'inscrit dans une période de rapports tendus, empreints de multiples ambiguités, entre les organisations indiennes et le pouvoir en place depuis 2006.

\section{Démocratie participative et État plurinational : une construction précaire}

On observe ainsi un fort décalage entre les changements constitutionnels et le poids restreint des acteurs indiens dans le changement politique ou le choix du modèle de développement économique ; changements dont ils semblent avoir somme toute peu bénéficié. Ce paradoxe n'est pourtant pas propre à l'Équateur ${ }^{60}$.

Le rôle des mouvements indiens dans le processus de démocratisation en Équateur ces vingt dernières années s'avère crucial à maints égards : ceuxci remettent en cause les fondements de la démocratie représentative et ceux de l'État-nation, et promeuvent une reconfiguration des modalités de représentation et participation politique à diverses échelles, du local au national ; ils obtiennent des droits collectifs et participent à la formulation des politiques publiques majeures ; enfin ils revendiquent la construction d'un État plurinational fondé sur la reconnaissance politique et la représentation de groupes dotés d'une identité culturelle. En bref c'est toute l'organisation politique, sociale et économique de la Nation qui est en jeu. La revendication d'une démocratie participative, couplée à celle d'État plurinational, constitue le cœur du projet politique indien.

59. Les récentes élections présidentielle et législative (17 février 2013) confirment ce déclin, puisque le candidat de l'Alliance « Unité Plurinationale des Gauches » (dont fait partie Pachakutik), A. Acosta, obtient à peine $3.2 \%$ et le mouvement n'obtient que 6 députés sur 137 soit $4 \%$ de la représentation législative. Correa est réélu avec $57 \%$ des voix et son parti domine le Congrès (90 sièges sur 137).

60. Un débat oppose les partisans du multiculturalisme institutionnel, conçu comme un outil d'émancipation des groupes indiens reconnus dans leur différence et intégrés politiquement, et ceux qui jugent ce multiculturalisme trompeur voire illusoire, considérant qu'il n' affecte pas fondamentalement les rapports de pouvoir sociaux et politiques traditionnels. Pour une synthèse de ce débat : LOPEZ CABALLERO P., "Altérités intimes, altérités éloignées : la greffe du multiculturalisme en Amérique latine », in Critique internationale, vol. 2, n51, 2011p. 129-149 ; CERVONE E., « Los desafíos del multiculturalismo », in MARTINEZ C. (ed.), op. cit., 2010, p. 199-214. 
Sur le plan théorique, l'apport majeur concerne le thème de la représentation et de la représentativité, puisqu'ils dénient à la démocratie représentative toute légitimité. Ce sont bel et bien l'intégration à la communauté politique et l'accès à la citoyenneté qui sont en débat : cette intégration ne devrait plus s'effectuer uniquement sous la forme libérale individuelle, mais devrait aussi inclure des groupes ( «peuples et nationalités ») fondés sur une identité culturelle. Groupes dont la source de légitimité majeure est le degré de mobilisation atteint par les organisations indiennes qui les représentent. Il ne s'agit nullement de se cantonner à une autonomie locale des peuples indiens au sein d'un territoire restreint ou à de simples mesures de reconnaissance de spécificités culturelles (éducation bilingue), mais bien d'obtenir une réorientation des modalités de la représentation. Celle-ci n'est pas effective au niveau national, mais se concrétise au niveau local et provincial. Ainsi, la reconnaissance des « groupes indiens » facilite une reconnaissance effective des « individus » : ce qui n'a pu être conquis en tant qu'individus auparavant, ou ne l'a été que formellement avec la transition démocratique, à savoir le respect de leur culture, mais aussi « de leur égalité citoyenne en tant qu'individus », peut être conquis avec la mobilisation puis la reconnaissance officielle des peuples indiens. L'identité culturelle devient le support de l'identité individuelle, et en cela, la position indienne coïncide avec la perspective multiculturaliste et communautarienne. Par là même, la citoyenneté ethnique ou différenciée, prônée par W. Kymlicka comme une modalité complémentaire à la citoyenneté nationale afin de revaloriser cette dernière, a sans aucun doute gagné en légitimité depuis vingt ans, dans le discours indien et la société équatorienne.

On assiste donc en Équateur à la construction encore fragile d'une démocratie locale fondée sur un mélange de représentation et de participation des communautés et organisations indiennes à différentes instances de délibération. Le débat politique est plus ouvert aux revendications indiennes qui ont acquis droit de cité via deux Constitutions. Des efforts notoires ont été faits pour redistribuer le pouvoir ; le thème des autonomies indiennes a progressé, non sans résistances, dans l'organisation administrative et territoriale. La promotion d'un discours d'égalité et de refus des discriminations et d'une politique favorable aux conditions de vie des communautés indiennes a aussi vu le jour au niveau national. Les acquis sont donc essentiels. Mais on ne saurait sous-estimer les obstacles à la mise en œuvre des nouveaux droits collectifs dans le projet de développement économique du gouvernement Correa, certes à vocation souverainiste, mais reposant sur un modèle « néo-extractiviste », axé principalement sur la transformation des matières premières. Un dialogue de sourds persiste donc entre, d'une part, un mouvement indien qui souhaite faire admettre, au sein de la société tout entière, la portée de «l'État plurinational » dans la vie quotidienne, et d'autre part 
un gouvernement qui se veut le représentant et héraut d'une «Nation en refondation » et dénonce les particularismes.

Le projet indien d'État plurinational et de construction d'une démocratie effective « décolonisée » montre que le changement institutionnel, principal axe du changement politique depuis 1990, est insuffisant. L'enjeu central est désormais la mise en cause du modèle d'appropriation, de gestion et d'usage des ressources naturelles, principal motif du conflit politique dans la région andine et les pays latino-américains, confrontés au boom de l'exploitation minière. La mise en œuvre des droits collectifs et de l'État plurinational se heurte donc plus que jamais à de profonds défis. 